\title{
A NEW METHOD FOR THE MECHANICAL ANALYSIS OF SOILS AND OTHER DISPERSIONS.
}

\author{
By GILBERT WOODING ROBINSON, M.A. \\ Adviser in Agricultural Chemistry, University College \\ of North Wales, Bangor.
}

INTRODUCTION.

Atrention has been given of recent years to the possibility of devising methods of mechanical analysis which shall express the mechanical composition of a soil or clay by a continuous curve. The standard methods of mechanical analysis, as for example that followed in England, can of course be used to obtain such curves, but with the comparatively small number of fractions separated, very little detail can be inserted. Any multiplication in the number of fractions must inevitably be attended by an increase of experimental errors, and the problem must therefore be attacked by other methods.

Odén has devised an elegant method whereby the mechanical analysis of a soil or clay can be derived from an experimental curve showing the rate of accumulation of sediment from a column of material in suspension ${ }^{1}$. The weight of material is automatically registered from time to time and from the curve obtained a mass distribution curve is derived which gives a detailed representation of the composition of the material under examination. Wiegner ${ }^{2}$ has applied the theoretical principles of the Odén method in a very simple apparatus consisting essentially of a U-tube system in which a column of sedimenting suspension is balanced against a column of pure water. As the material falls below. the point at which the water tube joins the fall tube, the specific gravity of the suspension decreases, resulting in a corresponding fall in the water column. The height of the water column is read from time to time and

1 Int. Mitt. Bodenkunde, 1915, 5, 257-311; Koll. Zeit. 1916, 18, 33-48; Trans. Faraday Soc. 1922, 17, 327-348: Nefedof, J. Exp. Landw. 1902, 3, 421-449, outlines a method similar in principle to that of Odén, but apparently purely empirical.

2 Landro. Versuchs Stat. 1918, 91, 41. 
by appropriate calculation the experimental results can be thrown into the form of curves similar to those obtained by Oden's instrument. Both Odén's and Wiegner's methods have found applications in the study of dispersions ${ }^{1}$.

A serious drawback to Oden's apparatus is its expense. While it is of the highest value for the critical investigation of comparatively small numbers of samples, it can hardly come into use for routine work as the resources of an ordinary provincial institution would scarcely be equal to the outlay involved in setting up more than one such instrument and only comparatively small numbers of soils could be dealt with. Wiegner's apparatus is cheaper and could by modifications ${ }^{2}$ be made to give results of considerable accuracy. But here the time factor is serious. Using, as is necessary, a column about a metre long, several days would be required to obtain information as to the finer fractions which exert such an important effect on the properties of the soil.

In the present work the writer has attempted to devise a method capable of giving more detailed data than the ordinary sedimentation methods and which, though not giving continuous curves, avoids the drawbacks of the Odén and. Wiegner methods and can be used for standard mechanical analysis with great saving of time.

Before discussing the new method of mechanical analysis a few remarks may be made on the expression of mechanical composition by means of curves. The simplest method of expressing the results of a mechanical analysis is to plot summation percentages ${ }^{3}$ against particle sizes. Such a method is however almost useless in the case of highly dispersed substances, because in order to show the complete range the most characteristic particle sizes are cramped together near the $y$-axis. A better distribution will be obtained by using the logarithms of particle sizes. The end of the curve corresponding to zero size is of course at $-\infty$ but in practice a very manageable type of curve will be obtained.

Since the separation of particles of diameter smaller than $.2 \mathrm{~mm}$. is universally based on the principle of subsidence, whereby fractions are distinguished by their different settling velocities in water, there are good reasons for using logarithms of settling velocities instead of logarithms of particle sizes. In the ensuing treatment this method is used, the velocities being calculated in ordinary c.G.s. units. The same

1 Koll. Zeit. 1920, 26, 100-121; ibid. 1920, 26, 121-138. J. Landwo. 1921, 69, 5-32.

2 As for instance in the method of reading the height of the water column.

- i.e. percentages of material of a given particle size or smaller.

- Cf. Whittles, J. Agric. Sci. 1822, 12, 166-181. 


\section{Mechanical Analysis of Soils and other Dispersions}

type of curve will be given and it will dispense with any assumption as to the size, shape and density of the settling particles.

In this connexion, the probable effect of the gel coating which has been postulated for the finer particles of a soil or clay on the sedimentation of such material is worthy of consideration. It has been suggested that such coatings are not to be considered as uniform and discrete but as concentric shells of increasing degree of hydration. As an approximate basis of calculation let us assume that the emulsoid coating has the same density as the water in which the particles are suspended. What will be the effect of this coating on the settling velocity? If $a$ be the radius of the falling particle and $d$ the thickness of the gel coating or shell, then the velocity of fall of the particle in the absence of any coating is given by

$$
v_{1}=\frac{2}{9} \cdot \frac{g a^{2}}{\eta} \cdot(\rho-1)
$$

using the usual notation and assuming the density of water $=1$, and the velocity of the coated particle by

$$
v_{2}=\frac{2}{9} \cdot \frac{g(a+d)^{2}}{\eta} \cdot\left(\rho_{1}-1\right)
$$

where $p_{1}$ is the mean density of the coated particle.

Now

$$
\begin{aligned}
\rho_{1} & =\frac{\text { weight of particle }+ \text { shell }}{\text { volume }} \\
& =\frac{\frac{4}{3} \pi a^{3} \rho+\frac{4}{3} \pi\left[(a+d)^{3}-a^{3}\right]}{\frac{4}{3} \pi(a+d)^{3}} \\
& =\frac{a^{3} \rho+\left[(a+d)^{3}-a^{3}\right]}{(a+d)^{3}} .
\end{aligned}
$$

Then

$$
\begin{aligned}
v_{2} & =\frac{2}{9} \cdot \frac{g(a+d)^{2}}{\eta} \cdot\left\{\frac{a^{3} \rho+\left[(a+d)^{3}-a^{3}\right]}{(a+d)^{3}}-1\right\} \\
& =\frac{2}{9} \cdot \frac{g}{\eta} \cdot \frac{a^{3}(\rho-1)}{a+d}, \\
\therefore \frac{v_{1}}{v_{2}} & =\frac{a+d}{a}
\end{aligned}
$$

or

$$
v_{2}=v_{1} \cdot \frac{a}{a+d} \text {. }
$$

It will be seen that the presence of a coating of approximately the same density as the suspension medium will have a marked effect on the velocity of fall. The low velocities corresponding to the finer fractions 
will thus be conditioned not simply by the actual particle sizes but by the magnitude of the gel coating. The writer hopes to return to this point in a later paper. In the meantime it is suggested that the left hand portion of the curve in the case of clay soils may relate to such coated particles rather than to particles bounded by a solid surface. By using $\log v$ instead of $\log$ particle size, the necessity for a decision as to the significance of these small velocities is postponed.

It is of course obvious that the expression of the mechanical composition of a soil or clay by a curve of this type offers a way out of the appalling confusion created by the diversity of conventions used in different countries. Since practically all the methods in use are based on the principle of sedimentation, curves can easily be obtained if the settling velocities are known. Whether the principle is applicable to methods in which separation is effected by currents of water of varying velocity, as in the Hilgard method, the writer is unable to decide. As a first approximation, it would appear that the method is applicable.

We have assumed that the viscosity coefficient in the Stokes' equation is constant. With varying temperatures this is of course not the case. This difficulty can however easily be solved if a standard temperature be adopted, say, $15^{\circ} \mathrm{C}$. By putting the viscosity coefficient of water at that temperature equal to unity and calculating the viscosities at other temperatures ${ }^{1}$ with reference to this, the correction can be applied. Thus if results obtained at varying temperature are to be compared, it will be necessary to use $\log (v \times$ specific viscosity) instead of $\log v$.

\section{Theory of New Method.}

A fundamental assumption underlying all methods of mechanical analysis by sedimentation is that particles in a column settle independently of each other. That there are limits to this assumption is obvious. According to Odén this condition is fulfilled in suspensions of concentration not greater than 1 per cent. ${ }^{2}$ Wiegner, on the other hand, brings evidence to show that concentrations of more than 5 per cent. can be used witbout serious inaccuracy, which may be due to the dangerous principle of compensating errors.

Let us assume a suspension of soil or other granular material to consist of a number of fractions, $a, b$, $c$, etc., each uniform in itself, having limiting velocities, $v_{1}, v_{2}, v_{3}$, etc., respectively, and present in

1 For the effect of temperature on the riscosity coefficient of water, see Hosking, Phil. Mag. 1907, 17, 509; ibid. 1909, 18, 260.

2 Int. Mitt. Bodenlunde, 1915, 5, 276. 


\section{Mechanical Analysis of Soils and other Dispersions}

concentrations, $A_{1}, A_{2}, A_{3}$, etc., respectively such that $\Sigma A=C^{1}$, the total concentration. Then if the fractions settle independently of each other, each fraction will behave as a separate column uniform in concentration from top to bottom and we may represent the state of affairs at the beginning of sedimentation as in the upper diagram of Fig. 1, the relative amount or partial concentration of each fraction being represented graphically by the thickness of its column on the diagram.

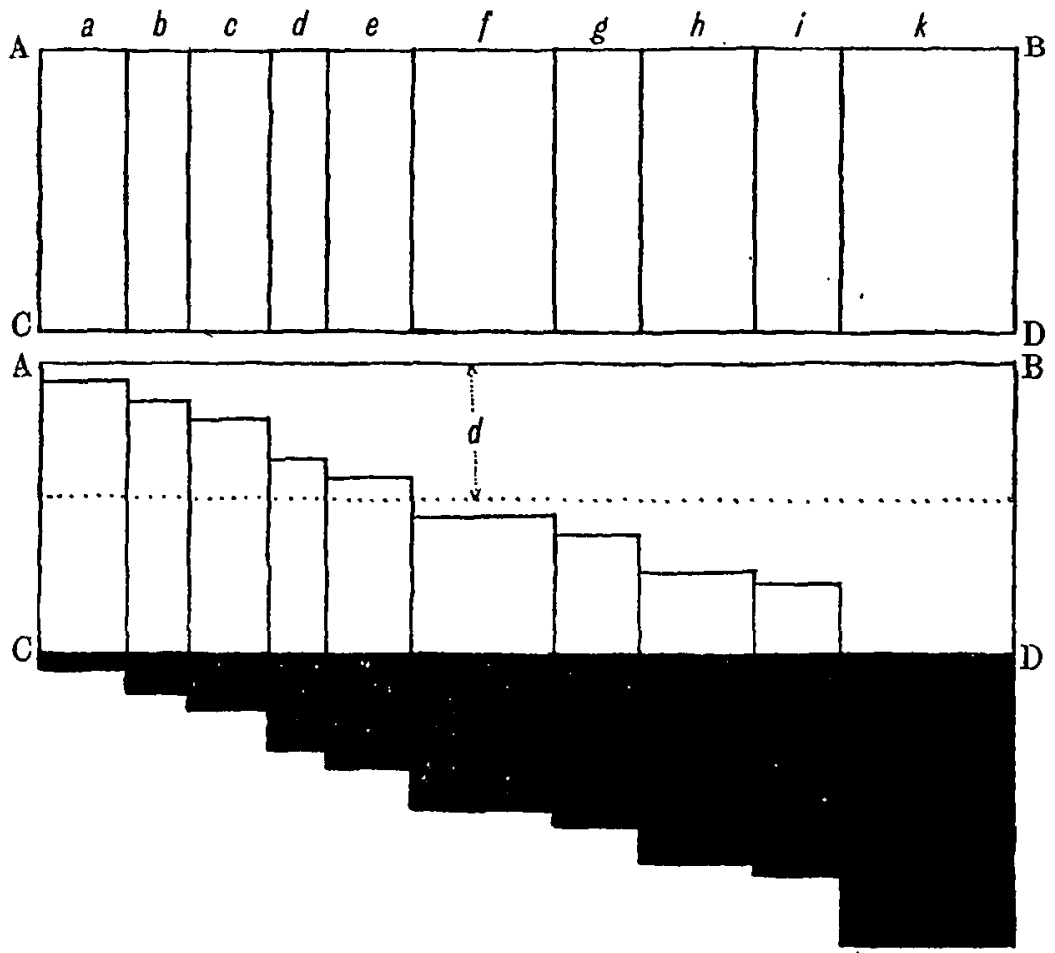

Fig. L. Diagrammatic representation of sedimentation.

As sedimentation proceeds each column will fall bodily at its own appropriate velocity and the disposition after settling has proceeded for a certain time may be represented by the lower figure of the diagram. The black portion below the line $C D$ will represent the amount of each fraction accumulated on the bottom of the sedimenting vessel, while the concentration of the suspension at any depth will be given by the total width of columns at that depth. Thus at depth $d$, the concentration will be equal to the sum of the partial concentrations of the

1 Or $\Sigma A$ +organic matter $=C$, in the case of ordinary soils. 
fractions, $a$ to $e$, having velocities less than $d / t$. The ratio of the concentration at depth $d$ after time $t$ to the total concentration at the beginning of the experiment will thus give the proportion of material having velocities less than $d / t$. By determining the concentration for different values of $d / t$ the data are obtained for a summation curve showing the relation between percentage of material and log settling velocity.

\section{EXPERIMENTAL.}

The method used consists in allowing a soil suspension of known concentration to settle in a cylindrical vessel and withdrawing samples for appropriate values of depth/time. By suitable choice of depth and time the concentration and hence the percentage of particles corresponding to any desired velocity can be obtained. Generally speaking a litre measuring cylinder about $40 \mathrm{~cm}$. in height and $6 \mathrm{~cm}$. in diameter is used. There is of course no necessity to use a graduated vessel: any cylinder of uniform cross section and suitable dimensions may be used. Sampling of the suspension is carried out by means of a 20 c.c. pipette passed through a cork or shive and adjusted so that when the cork rests on the top of the cylinder the point of the pipette is at the desired depth below the surface of the liquid (see Fig. 2). The column having settled for the required time the pipette, previously adjusted for depth, is closed at the top with the finger, in order to avoid sampling the upper layers, and lowered very carefully till the cork rests on the top of the cylinder. The finger is then removed and 20 c.c. of the suspension withdrawn. Every precaution is of course taken to avoid shaking or mixing the layers of the suspension at the point of sampling. With a column of the dimensions mentioned the withdrawal of 20 c.c. causes a fall in level of about $7 \mathrm{~mm}$. This probably represents the extreme error

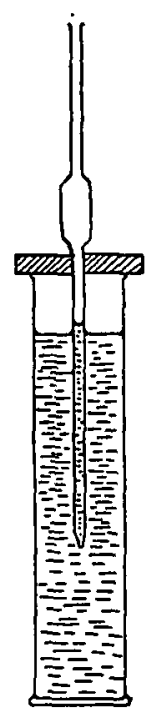

Fig. 2. Method of sampling. in sampling. It is assumed that the 20 c.c. of suspension withdrawn represents the concentration at the point of the pipette. Probably the liquid comes mainly from above, but to some extent from below this point. A separate experiment with a column which had settled for several weeks and which had formed clearly defined strata, showed that it was possible to pipette to within 2 to $3 \mathrm{~mm}$. of a stratum without disturbance. It will be shown later that an error of a few millimetres in sampling involves a negligible error in the final result. Careful manipulation is of course necessary in this operation. The 20 c.c. of suspension 


\section{Mechanical Analysis of Soils and other Dispersions}

is delivered into a flat porcelain dish which has been previously ignited and weighed. Dishes ordinarily used for the estimation of total solids in milk are convenient for the purpose. The sample is taken to dryness on the water bath and, if the estimation is to be made on unignited material, weighed after attaining constant weight. Ordinarily it is ignited in a muffle, an operation which only takes a few minutes at red heat, and weighed after cooling in a desiccator. From the weight of ignited material the concentration of the sample of suspension is calculated. By sampling in such a way that successively smaller values of depth/time are used, the same suspension may be shaken up and sampled over and over again. The partial concentration of any fraction at a given depth is unaltered until the top. of the fraction column has sunk below that depth, as will be seen by reference to Fig. 1. The removal of a sample of suspension does not therefore affect the concentration of the suspension with respect to fractions of smaller velocities.

In an actual experiment a 2.5 per cent. suspension of a clay was prepared by shaking up 100 grams of powdered clay for 24 hours with 2 litres of water containing 100 c.c. of 1 per cent. sodium carbonate solution and making up finally to 4 litres. A litre cylinder was then filled to within 3-4 cm. of the top with the well mixed suspension and after again shaking for a minute, allowed to stand for six minutes. A 20 c.c. sample was then withdrawn at $36 \mathrm{~cm}$. depth as described above. After drying and ignition, the weight of ignited material was found to be $\cdot 376$ gram. Subtracting 005 gram for the amount of sodium carbonate in 20 c.c., we have the nett weight of ignited material as $\cdot 371$ gram and the concentration of the suspension at the point sampled, 1.855 per cent. The original concentration being 2.5 per cent., we find that the concentration of the suspension at $36 \mathrm{~cm}$. after six minutes is $\frac{1.855}{2.5} \times 100=74.2$ per cent. of the original concentration. In other words 74.2 per cent. of the clay, reckoned as ignited material, has a settling velocity less than $\cdot 1 \mathrm{~cm} . / \mathrm{sec}$. Other determinations were made for successively smaller velocities and the results are set out in Table $I$. For the sake of brevity the weight of ignited material is given after subtraction of the $\cdot 005$ gram of sodium carbonate.

In Fig. 3 curves are shown for the clay of the experiment just described and for a few other typical clays and soils. The vertical dotted lines, $A, B$, and $C$, are the ordinates corresponding to clay, fine silt, and silt, respectively, according to their settling velocities in the English method. In order to bring the fine sand, coarse sand and fine gravel 
Table I. London Clay. 2.5 per cent. suspension in $\cdot 025$ per cent. sodium carbonate solution. Temperature $12-16^{\circ}$.

\begin{tabular}{|c|c|c|c|c|c|c|}
\hline $\begin{array}{l}\text { Time } \\
\text { secs }\end{array}$ & $\begin{array}{l}\text { Depth } \\
\text { cms }\end{array}$ & $\begin{array}{l}\text { Velocity } \\
\mathrm{cm} / \mathrm{sec}\end{array}$ & $\log v$ & $\begin{array}{c}\text { Ignited } \\
\text { materal } \\
\text { in } 20 \mathrm{cc} \\
\text { gms }\end{array}$ & $\begin{array}{c}\text { Concen- } \\
\text { tration } \\
\%\end{array}$ & $\begin{array}{l}\% \text { of } \\
\text { ongunal } \\
\text { concen } \\
\text { tration }\end{array}$ \\
\hline $\begin{array}{r}360 \\
600 \\
600 \\
6000 \\
6000 \\
60000 \\
60000 \\
600000 \\
600000\end{array}$ & $\begin{array}{r}36 \\
20 \\
6 \\
20 \\
6 \\
20 \\
6 \\
20 \\
6\end{array}$ & $\begin{array}{l}1000 \\
0333 \\
0100 \\
0033 \\
0010 \\
00033 \\
00010 \\
000033 \\
000010\end{array}$ & 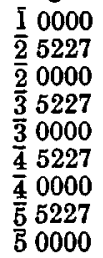 & $\begin{array}{l}0371 \\
0358 \\
0325 \\
0293 \\
0252 \\
0 \quad 220 \\
0178 \\
0135 \\
0093\end{array}$ & $\begin{array}{ll}1 & 855 \\
1 & 790 \\
1 & 625 \\
1 & 465 \\
1 & 260 \\
1 & 100 \\
0 & 890 \\
0 & 675 \\
0 & 465\end{array}$ & $\begin{array}{l}742 \\
716 \\
650 \\
586 \\
504 \\
440 \\
356 \\
270 \\
1865\end{array}$ \\
\hline
\end{tabular}

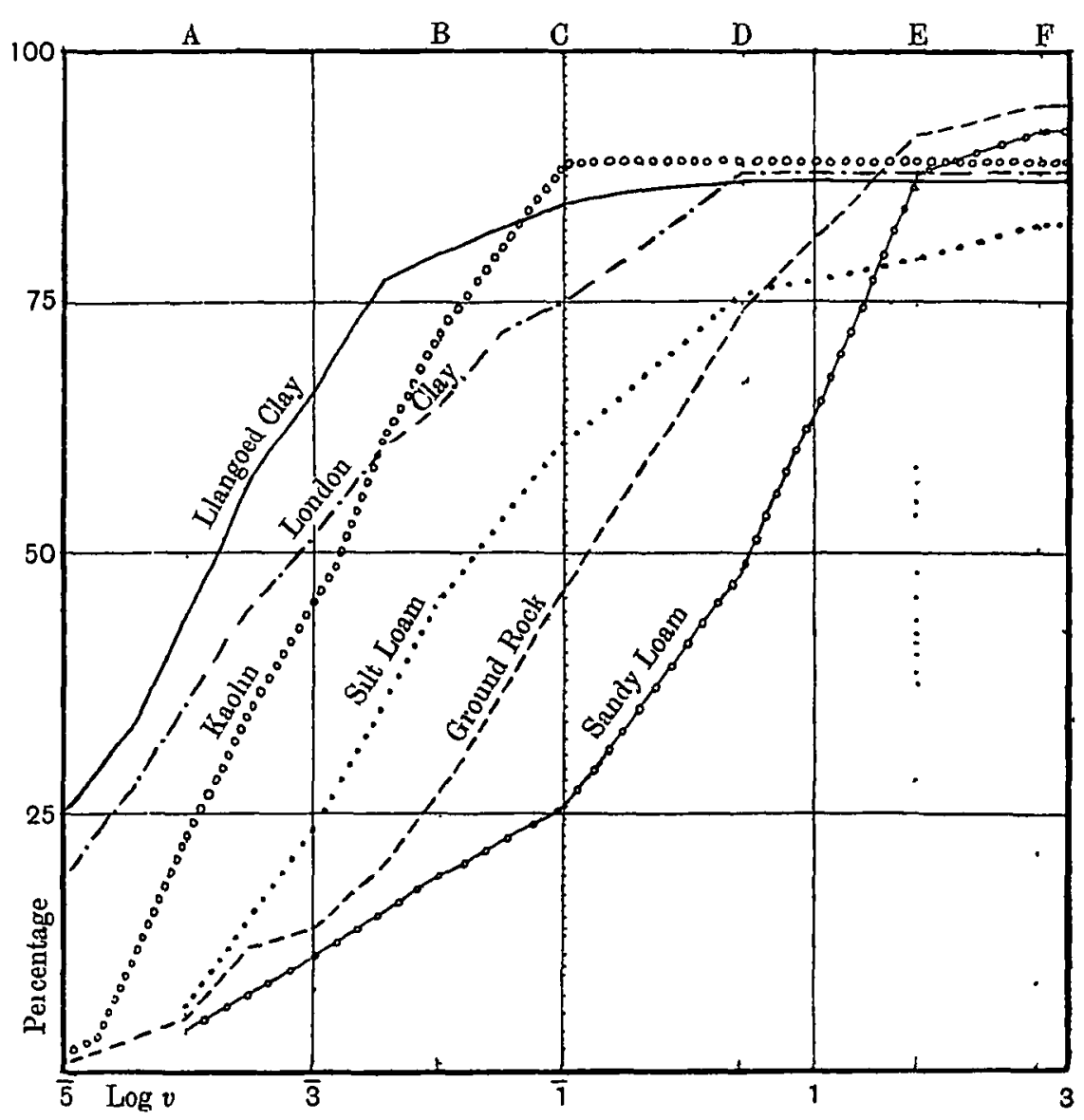

Fig. 3 Summation Curves showng Composition of Typical Souls and Clays. 


\section{Mechanical Analysis of Soils and other Dispersions}

into the diagram, their velocity values were calculated on the assumption that they obey Stokes' law and that the diameter of particle represented by the upper limit of the silt is $.04 \mathrm{~mm}$. The lines thus obtained are $D, E$, and $F$, respectively and are inserted for the sake of completeness. By taking into account the coefficient of viscosity and using glycerine-water mixtures, these points on the distribution curve might be obtained by the above method. It is however simpler to use the sieve method to fill in the right hand portion of the curve. It may be added that the data of an ordinary mechanical analysis are given graphically by the difference between successive intercepts on the ordinates $A, B, C$, etc. For example the fine silt is given by the difference between the intercept on $B$ and the intercept on $A$, the silt by the difference between the intercept on $C$ and the intercept on $B$ and so on.

By suitable choice of depths and times any required degree of detail can be secured in any part of the curve. If a large number of points are required it is convenient to prepare a large volume of suspension and work with a number of separate cylinders. The details may be left to the convenience and ingenuity of individual operators. Possibly a more convenient, though scarcely less expensive, method of sampling may be devised. Any measurable physical property of dispersions which depends on concentration may be considered in this connexion. For very dilute suspensions it is possible that a nephelometric method might be devised.

The method may find its best use as a substitute for the present standard method of mechanical analysis. The suggested procedure in this case is as follows. The air dried sample is treated with $N / 5^{\circ}$ hydrochloric acid exactly as in the ordinary method, using however 20 grams instead of 10 grams of soil. The fine gravel and coarse sand are separated in the ordinary way and the finer material passing the 100 mesh sieve is shaken with $600-700$ c.c. of water and 50 c.c. of 10 per cent. ammonia in an end over end shaker for two to four hours, the longer period being necessary in the case of soils with much organic matter. After shaking, the suspension is made up to one litre, which is equivalent to 2 per cent., reckoning on the original material. The following determinations are then made successively by the method described above.

\begin{tabular}{|c|c|c|c|}
\hline $\begin{array}{l}\text { Depth } \\
\mathrm{cm} .\end{array}$ & \begin{tabular}{l}
\multicolumn{2}{c}{ Time } \\
hrs. min.
\end{tabular} & $\begin{array}{l}\text { Velocity } \\
\mathrm{cm} . / \mathrm{sec} .\end{array}$ & $\begin{array}{c}\text { Giving } \\
\text { percentage of }\end{array}$ \\
\hline $\begin{array}{ll}\text { (a) } & 30 \\
\text { (b) } & 12\end{array}$ & $\begin{array}{rc}0 & 5 \\
0 & 20 \\
16 & 40\end{array}$ & $\begin{array}{l}0.1 \\
0.01 \\
0.0001\end{array}$ & $\begin{array}{r}\text { silt }+ \text { fine silt }+ \text { clay } \\
\text { fine silt }+ \text { clay } \\
\text { clay }\end{array}$ \\
\hline $\begin{array}{ll}\text { (c) } & 6 \\
\text { or } & 7 \cdot 2\end{array}$ & $\begin{array}{l}16 \\
20\end{array}$ & 0.0001 & \\
\hline or 8.6 & 24 & & \\
\hline
\end{tabular}


Lastly to determine the fine sand, the suspension remaining after the last sampling is poured away to about 200 c.c. without shaking up the sediment. The remaining suspension and sediment are then washed into a beaker and the fine sand determined by the ordinary method, using the $10 \mathrm{~cm}$. and 100 seconds, or $7.5 \mathrm{~cm}$. and 75 seconds sedimentation.

The following figures are the results of an actual determination on a Pennant Grit soil from Glamorganshire:

$\begin{array}{ccccc}\text { By ordinary method } & \text { Fine gravel } & \ldots & \ldots & 4 \cdot 6 \% \\ & \text { Coarse sand } & \ldots & \ldots & 20 \cdot 1 \\ \text { Moisture } & \ldots & \ldots & 4 \cdot 2 \\ \text { Organic matter } & \ldots & \ldots & \frac{10 \cdot 2}{39 \cdot 1} \\ & \text { Total } & \ldots & \ldots & 39 \cdot 1\end{array}$

So that fine sand + silt + fine silt + clay should $=100-39 \cdot 1=60 \cdot 9$

By new method, using $2 \%$ suspension.

For 5 mins. and $30 \mathrm{~cm}$., weight of ignited material in 20 c.c. $=\cdot 112 \mathrm{gm}$.

Therefore concentration $=5 \times \cdot 112=.560 \%$.

Therefore silt + fine silt + clay $=\frac{.560 \times 100}{2}=28.0 \%$

Similarly for 20 mins. and $12 \mathrm{~cm}$, ignited material $=.065 \mathrm{gm}$.

Concentration $=\cdot 325 \%$ and fine silt + clay $=\frac{.325 \times 100}{2}=16.25 \%$

Similarly for $20 \mathrm{hrs}$. and $7 \cdot 2 \mathrm{~cm}$. ignited material $=.019 \mathrm{gm}$.

Concentration $=.095 \%$ and clay $=\frac{.095 \times 100}{2}=4.75 \%$

Subtracting (4) from (3), fine silt $=11.5 \%$.

Subtracting (3) from (2), silt $=11.75 \%$.

Subtracting ( 2 ) from (1) fine sand $=32.9 \%$.

By direct sedimentation at end of experiment, fine sand $=32.5 \%$.

\section{Effect of Variations in Conditions of Working.}

In order to form some idea as to the latitude allowable in the conditions of working the following points were investigated.

(a) Effect of concentration. A number of determinations were made on a clay suspension of varying concentrations. Results are given for different times and depths.

\begin{tabular}{|c|c|c|}
\hline & $\begin{array}{l}\text { entration } \\
\text { original } \\
\text { pension }\end{array}$ & $\begin{array}{l}\text { Percentage } \\
\text { of original } \\
\text { concentration }\end{array}$ \\
\hline $\begin{array}{l}\text { Depth } 6 \mathrm{~cm} \text {. } \\
\text { Time } 10 \text { mins. }\end{array}$ & $\left\{\begin{array}{l}.50 \% \\
.625 \\
1.00 \\
2.00 \\
2.50 \\
4.00 \\
5.00\end{array}\right.$ & $\begin{array}{l}63 \cdot 0 \\
61 \cdot 0 \\
62 \cdot 0 \\
63 \cdot 2 \\
64 \cdot 0 \\
63 \cdot 1 \\
65 \cdot 7\end{array}$ \\
\hline
\end{tabular}




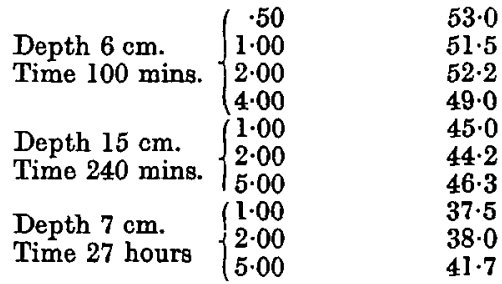

The variations in the results obtained with varying concentrations are not serious when the nature of mechanical analysis and the nature of the material under examination is taken into account. In the standard English method the concentration used is about 2 per cent. In view of the small weights to be dealt with in very dilute suspensions and the consequent magnification of weighing errors, it was decided to use 2 per cent. suspensions as a general rule and in the comparisons given later of the results by the new method with those by the old method, this concentration was used.

(b) Diameter of column. The diameter of the column may be expected to have some effect on the result obtained since, apart from any boundary effect, the fall in height due to the removal of liquid will be greater in narrow columns. The following experiment serves to test this point.

$\begin{array}{cc}\text { Clay suspension } 2.5 \% & \text { Time } 19.5 \mathrm{hrs} . \text { Depth } 9 \mathrm{~cm} . \\ \text { Diameter of cylinder } & \text { Weight of ignited material } \\ 7.7 \mathrm{~cm} . & \cdot 102 \mathrm{gm} . \\ 6.0 & .101 \\ 4.6 & .102 \\ 3.4 & .098\end{array}$

The last cylinder was an ordinary 250 c.c. measuring cylinder. It would appear that with $\frac{1}{2}$ litre, litre and 2 litre cylinders consistent results may be obtained and that the diameter of the cylinder is immaterial. In general, a litre or $\frac{1}{2}$ litre cylinder was used.

(c) Equivalence of Depth/Time ratio. Theoretically the same concentration should be obtained for different times and depths provided that the ratio depth/time, i.e. the limiting velocity is constant. This point is investigated in the following experiments.

Kaolin. $2 \cdot 5 \%$ suspension in $025 \%$ sodium carbonate.

$\begin{array}{ccc}\text { Depth } & \text { Time } & \text { Weight of ignited } \\ \text { material } \\ \mathbf{5} & \text { hours } & \text { gm. } \\ 19.5 & 5 & .165 \\ & 19.5 & .169\end{array}$

Clay. $2.5 \%$ suspension in $.025 \%$ sodium carbonate.

$\begin{array}{rll}4 & 1 & .254 \\ 12 & 3 & .253 \\ 5 & 18 \cdot 75 & \cdot 178 \\ 18 & 68 & \cdot 177\end{array}$


(d) Errors in Depth of Sampling. The effect of errors in depth of sampling may be best demonstrated by considering the nature of the vertical concentration gradients in a column of suspension after varying times. A series of curves showing the relation between depth and concentration for different times can readily be derived from the summation curve for the material under consideration. For example if it be known from the summation curve that 50 per cent. of the material has

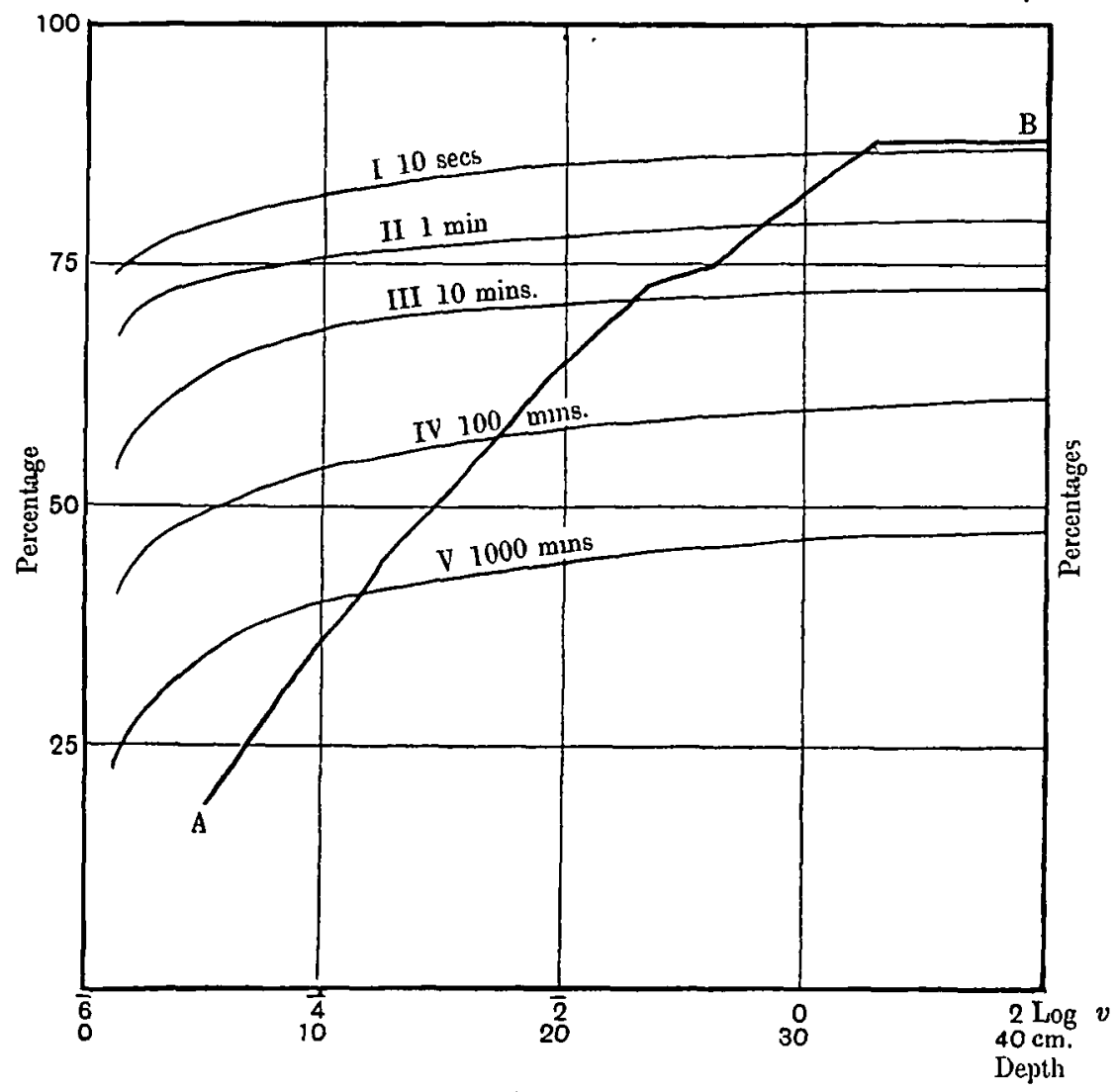

Fig. 4. Concentration Gradients at Different Depths.

a limiting velocity less than $\cdot 01 \mathrm{~cm}$. per second $(\log v=\overline{2} \cdot 0000)$, then at depth $10 \mathrm{~cm}$. after 1000 seconds, the concentration will be 50 per cent. of the original concentration. A series of concentration gradients for a clay is shown in Fig. $4 . A B$ is the summation curve and the curves I, II, III, IV, and V give the percentages of the original concentration at different depths for 10 secs., 1 min., 10 mins., 100 mins., and 


\section{Mechanical Analysis of Soils and other Dispersions}

1000 mins. respectively. The $\log v$ abscissae refer of course to the summation curve $A B$, and the depth abscissae to the concentration curves.

It will be seen that the change in concentration with depth after any given time is very gradual below the first few centimetres. Thus, after ten minutes, the concentration in the case illustrated only changes about 2.5 per cent. of the total concentration between $10 \mathrm{~cm}$. and $20 \mathrm{~cm}$. With longer times the gradient becomes rather steeper. Errors of the order of a few millimetres in depth of sampling have thus very little effect on the concentration obtained. This of course only holds so long as the material under experiment has a fairly smooth summation curve. With a material having an irregular type of curve, depth errors might be more serious.

Similar considerations can be developed for the time and temperature error.

The errors introduced by the above variations in working conditions though scarcely negligible are nevertheless not serious when the character of the material is considered and it may be doubted whether they are of significance from the point of view of the genetics and physical properties of soils and clays. The Odén method is of course less exceptionable from the point of view of delicacy and must be used where critical data are required. The method described in the present paper could of course be made to give results strictly comparable among themselves. It is however desirable to have a method which can admit of some latitude to suit the convenience of individual workers and which has to that extent and within reasonable limits the character of an absolute method.

\section{Agreement with Results obtained by the Standard Method.}

In view of the enormous number of results accumulated by the older method, it would be a doubtful advance to suggest a new method, even though more convenient and accurate, if the results obtained by it could not be used for comparison with the older results. A considerable number of determinations were therefore made by the new method and the results compared with those already obtained by the old method. In the following table the results of this comparison are given. For convenience of statement only three values are given, namely, clay, fine silt, and silt. The same method of dispersion was used throughout except in the case of certain clays, which contained practically no organic matter and were dispersed in .025 per cent. sodium carbonate. 
Table II. Comparison of Results by Old and New Methods.

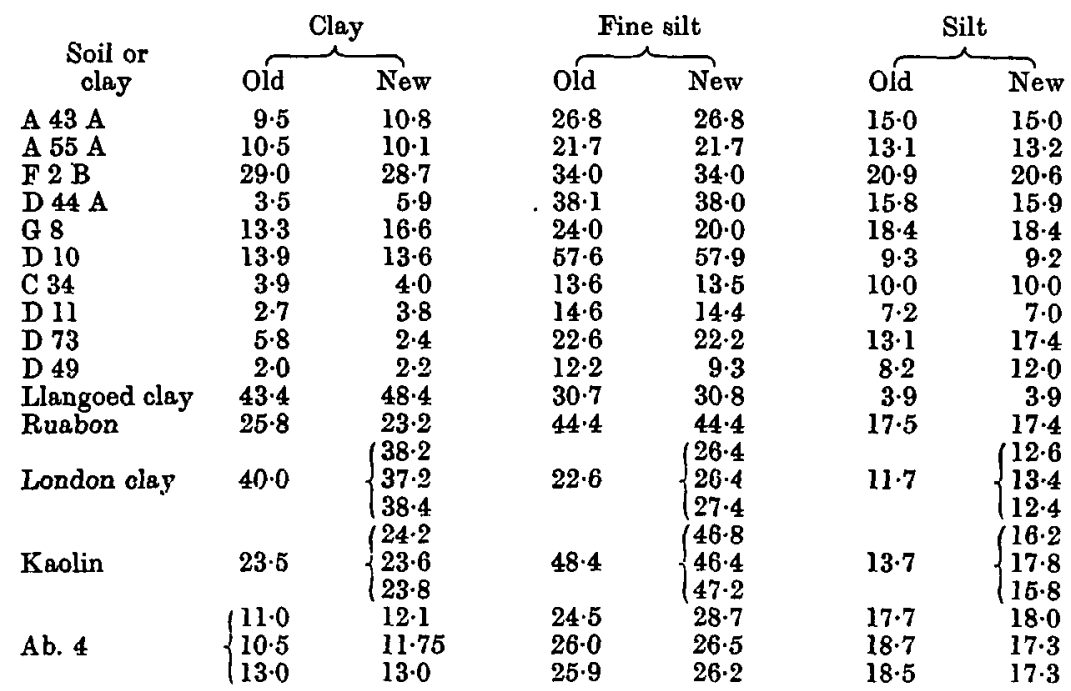

The agreement between the two series of results is generally close and not unsatisfactory when it is considered that mechanical analysis by the older method is liable to considerable errors. On the average, putting the figures obtained by the old method as 100 , the new method gives 102.7 for the clay, 100 for the fine silt and 99.6 for the silt. It would thus appear that there is no appreciable constant error in comparing results by the two methods. In view of the large number of manipulations required in the old method, there are more occasions for error than in the new, and any serious disagreement is at least as likely to be due to errors in the former as in the latter method.

It will be noticed that the most serious disagreements are in the case of light soils. To secure a perfect comparison it would be necessary to secure that the preliminary dispersion is exactly the same in both methods. In the standard method the material is repeatedly triturated after each pouring off. The method provisionally adopted for dispersion in the new method, namely a 2 to 4 hour shaking in an end over end shaker would appear to give a comparable degree of dispersion. The longer period is apparently necessary in the case of soils rich in organic matter. Dispersion by sodium carbonate ${ }^{1}$ gave good results with raw clays but was unsatisfactory with soils containing much organic matter.

+ Cf. Joseph and Martin, J. Agric. Sci. 1921, 11, 293-303. 


\section{Mechanical Analysis of Soils and other Dispersions}

\section{Conclusion.}

The method of mechanical analysis above described has two recommendations. It is more expeditious and economical than the standard method. Granted that the new method gives reliable results, its adoption would remove one of the gravest objections against the present method, namely its laboriousness. With the new method it has been found possible in the writer's laboratory to carry out six mechanical analyses in a day. With proper organisation there should be no difficulty in carrying out 35-40 analyses in a week. Anyone familiar with the routine of the older methods will realise that it would be impossible to deal with such numbers single handed. There is the further consideration of economy in the use of beakers, filters, etc., to say nothing of the distilled water, ammonia and hydrochloric acid required. On the other hand the suggested method requires careful manipulation. Working with 2 per cent. suspensions, an error of $1 \mathrm{mgm}$. in weighing corresponds to $\cdot 25$ per cent. in the result obtained. It is doubtful if the method would commend itself for teaching purposes.

The new method may be used within limits for obtaining continuous curves such as are obtained by the Odén and Wiegner methods. Provided temperature conditions are controlled it is easily possible to carry the distribution curve for a soil or clay considerably beyond the limits of clay as defined by the standard method, and the constitution of the finer fractions can thus be investigated over ranges hitherto scarcely explored. An application may also be found in the investigation of changes in degree of dispersion consequent on manuring, cultivation and season.

With regard to the errors of the method it may be remembered that the experiments recorded were carried out in ordinary measuring cylinders. These are rarely uniform in cross section. With perfectly cylindrical columns, no doubt, better results would be obtained. With regard to temperature effects, no attempt was made to secure rigid control of temperature conditions. As in the ordinary method, the settling took place in an ordinary laboratory with its unavoidable vicissitudes of temperature. Care was however taken to avoid the proximity of sources of heat. In certain cases where the settling columns were in the vicinity of radiating surfaces, unsatisfactory results were obtained. Generally speaking for the longer periods the columns were

1 A considerable number of clays have, in fact, been followed by this method as far as $\log v=\overrightarrow{7}$, which appears to be near the lower limit. 
put away in a large cupboard in a room without any burners or other sources of heat. The possibility of convection currents was thus avoided as far as possible.

By a suitable choice of depths and times the method could be readily adapted to systems of fractionation other than that in use in this country. In the American systems, where the numbers of fractions separated are large, the amount of work involved and the possibilities of error are serious. The above method might be of use in such cases. By setting out the results as summation curves the mechanical analyses by any system of grading could be obtained by interpolation.

\section{SUMmaRY.}

(1) The expression of mechanical composition by means of continuous curves is discussed. It is suggested that a convenient representation will be obtained by showing summation percentage as a function of the logarithm of settling velocity.

(2) The effect of a gel coating on the settling velocity of a particle is examined and it is shown that a reduction in velocity takes place which is a simple function of the thickness of the gel coating.

(3) A method is outlined by which the mechanical composition of a soil or clay is derived from determinations of the concentration of a settling suspension for different values of depth/time.

(4) A shortened method for mechanical analysis is described which gives results in good agreement with results obtained by the present standard method.

(5) The effect of various modifications in conditions of working is discussed.

(6) The nature of the concentration gradients in a settling column of a suspension is examined. It is shown that below the first few centimetres the change in concentration with depth is very gradual. 